\title{
Correction to: Influence of Guillotine Clearance on Cut-Edge Damage to Nonoriented Electrical Steel
}

Canming Wang, Qiusheng Yan, Jiabin Lu, and Xiaowei Zhang

\section{Correction to: JMEPEG (2020) 29:573-581 \\ https://doi.org/10.1007/s11665-019-04526-w}

The corresponding author has been changed from Canming Wang to Qiusheng Yan to ensure that any correspondence is received. The change is made with the agreement of all authors. Qiusheng Yan can be contacted at qsyan@gdut.edu.cn.
Publisher's Note Springer Nature remains neutral with regard to jurisdictional claims in published maps and institutional affiliations.

The original article can be found online at https://oi.org/10.1007/ s11665-019-04526-w.

Canming Wang, Qiusheng Yan, Jiabin Lu, and Xiaowei Zhang, School of Electromechanical Engineering, Guangdong University of Technology, Guangzhou 510006, China. Contact e-mails: 435938395@qq.com and qsyan@gdut.edu.cn. 